\title{
Elimination of measles by 2024: achievements and challenges
}

\author{
Sabrina Afrin ${ }^{1^{*}}$, Kazi Taib Mamun ${ }^{2}$, Nabeela Mahboob ${ }^{1}$, Hasina Iqbal ${ }^{1}$, Hasnatul Jannat ${ }^{1}$ \\ ${ }^{1}$ Department of Microbiology, Popular Medical College, Dhaka, Bangladesh; \\ ${ }^{2}$ Infectious Disease Division, icddr, b, Dhaka, Bangladesh
}

\begin{abstract}
Measles is an infectious agent of viral origin with exceedingly high rate of transmissibility contributing to very high morbidity and mortality rates especially among children. Although measles is extremely infectious, control strategies of this virus used to be recognized as one of the most successful public health interventions ever undertaken. However, despite being vaccine-preventable disease measles has encountered an enormous resurgence as the rate of measles vaccination has declined and in many countries vaccination targets remain unmet and measles continues to claim hundreds of thousands of lives each year. This review discusses the reasons of the re-emergence of measles, the present global and Bangladesh situation and strategies that have been undertaken to combat this killer disease to eliminate measles globally by the year 2024 .
\end{abstract}

IMC J Med Sci 2020; 14(1): 007. EPub date: 05 April 2020

\section{Introduction}

Measles is a highly contagious virus that can affect people of all ages although it is considered primarily as a childhood illness. Being a killer virus in the pre-vaccine era measles used to kill 2 to 3 million people annually worldwide [1]. The incidences of devastating complications and sequelae of the pre-vaccine era have been plummeted by the collaborative global vaccination initiatives. However, in spite of the availability of the safe, potent and cost-effective vaccine, the hard fought gains against measles are threatened now and the number of measles cases has soared in recent years. The causes of the outbreaks vary but the sub-optimal vaccine delivery is at the root of them as eliminating the last pockets of the unvaccinated residents is the hardest. In view of the resurgence of measles in many countries of the world, the cardinal question asked by many is whether it will be really possible to eliminate this disease globally by the year 2024. This review discusses the clinical spectrum of measles, global elimination strategy and the likelihood of the worldwide elimination of this disease.

\section{The virus}

Measles virus a highly contagious member of the Morbillivirus genus within the Paramyxoviridae family, is a spherical shaped, enveloped virus studded with virus-coded glycoproteins. The 15 kilobase encapsidated negative-sense singlestranded genomic RNA contains approximately 15,894 nucleotides [2,3]. The genome encodes eight proteins, two of which ( $\mathrm{V}$ and $\mathrm{C}$ ) are nonstructural proteins. Among the six structural proteins, phosphoprotein (P), large protein (L), and nucleoprotein $(\mathrm{N})$ form the nucleocapsid whereas, the hemagglutinin protein $(\mathrm{H})$, fusion protein $(F)$, and matrix protein $(M)$, together with lipids from the host cell membrane, form the viral envelope. The hemagglutinin $(\mathrm{H})$ establishes initial contact with a cellular receptor mediating receptor binding and the fusion protein (F) is one of the important components of the measles virus fusion machinery [4]. Membrane fusion is not only required for virus-to-cell entry but also executes multinucleated giant cell formation [5]. Although measles virus is serologically monotypic and antigenically stable, by analysis of the 
sequences of the nucleoprotein (N) and hemagglutinin $(\mathrm{H})$ genes 8 clades of measles virus (designated $\mathrm{A}$ through $\mathrm{H}$ ) have been identified and these have been divided into 22 genotypes and one proposed genotype. Clades B, C, D, G and $\mathrm{H}$ each contain multiple genotypes (B1 - 3, C1 - 2, D1 - 10, $G 1-3, H 1-2)$. Clades $A, E$ and $F$ each contain a single genotype $(A, E, F)$. Infection by any genotype induces life-long immunity against all genotypes. Notably, there are no known biological differences between viruses of different genotypes and no genotype has been associated with variability in transmissibility, greater virulence or persistence, likelihood of developing severe sequelae, sensitivity of laboratory diagnosis. However, some genotypes may be associated with specific geographic regions [6]. New genotypes are likely to be identified with the assistance of molecular epidemiological investigation of measles outbreaks globally. This enhanced surveillance may allow us to observe the change in virus genotypes over time in a particular region establishing epidemiological links between cases in geographically distinct clusters [7]. In addition, molecular characterization of measles virus is an important component for assessing the effectiveness of vaccination programs and surveillance systems designed to achieve the elimination of measles [8,9].

\section{Clinical spectrum and complications of measles}

After an incubation period of 8-12 days, measles begins with increasing fever $\left(39^{\circ} \mathrm{C}-40.5^{\circ} \mathrm{C}\right)$, cough, coryza, and conjunctivitis. However, unlike other features of prodromal stage the Koplik's spot is considered to be pathognomic for measles. Discrete maculopapular rash which begins from face and spreads gradually to chest, trunk and limbs is another significant clinical feature of acute measles infection.

A leading cause of childhood morbidity and mortality is the development of secondary infections after acute measles infection globally. The most devastating complications of measles include respiratory and central nervous systems. Measles virus is associated with pneumonia due to the virus itself, pneumonia due to secondary bacterial infection and giant cell pneumonia. Most importantly, four types of measles-induced encephalitis namely primary measles encephalitis, acute post-measles encephalitis, measles inclusion body encephalitis and subacute sclerosing panencephalitis endanger the people who have suffered from measles infection. Primary measles encephalitis is concurrent with measles infection which approximately affects 1$3 / 1000$ patients with measles infection. Although considerable gaps remain in the knowledge of the underlying mechanism of it, primary viral invasion of neurological cells followed by chemokine induction and lymphocytic infiltration might be a possible mechanism [10]. Mortality rate as high as $10-15 \%$ has been reported. In addition, permanent neurological damage lasts in $25 \%$ of patients [11]. The most frequent central nervous system (CNS) complication of measles virus is acute post-measles encephalomyelitis [12]. Being an autoimmune disease it is developed by molecular mimicry where a cross reactive myelin antibody induces the CNS dysfunction. About 1 child out of every 1,000 who get measles can develop acute post-measles encephalomyelitis [11]. Measles inclusion body encephalitis is a disease of the immunocompromised hosts principally the children of around six years who contracted measles infection within one year. Although mortality rate is $75 \%$ only supportive treatment exists. In addition, subacute sclerosing panencephalitis (SSPE) is a very rare, but fatal central nervous system disorder which may occur 5-15 years after measles infection. The underlying mechanism could be the capability of measles virus to persist in neurons as a defective variant when the immune system fails to eliminate measles virusinfected cells completely from the CNS [13]. In addition, the complication rates are increased by immune deficiency disorders, malnutrition and vitamin A deficiency [14].

Measles infection in pregnant women is associated with several adverse events including increased risk of hospitalization and pneumonia [15]. In addition, there are significant risks to the fetus including miscarriage, stillbirth, low birth weight, preterm delivery [16]. In areas of ongoing outbreaks where there is sustained transmission in compact and overcrowded communities, serologic testing for measles IgG can be considered in pregnant women without documented immunity to measles. Pregnant women with suspected measles exposure but without immunity should receive intravenous 
immunoglobulin (IVIG) treatment within 6 days of measles exposure. If serologic testing and obtaining results are not available in a timely manner, and measles exposure is suspected in a non-immune pregnant woman, the patient should receive measles IVIG $[17,18]$.

\section{Strategies undertaken to eliminate measles}

The first evidence of measles virus came into light in 1757 when Francis Home, a Scottish physician, demonstrated that measles is caused by an infectious agent in the blood of patients. Later in 1954, John F. Enders and Dr. Thomas C. Peebles succeeded in isolating measles from 13-year-old David Edmonston's blood. Before implementation of the National Measles Vaccination program in 1963, approximately 500,000 persons in the United States were reported to have had measles every year, of whom 500 died, 48,000 were hospitalized, and another 1,000 had permanent brain damage [19]. Elimination of measles from a country means the absence of endemic measles cases for a period of $\geq 12$ months in the presence of a high-quality epidemiological surveillance which is supported by a laboratory network. In 2000, a historic milestone was achieved by the USA as measles was declared to be eliminated there by the widespread use of safe and costeffective measles vaccine. Subsequently, however, vaccination rates had fallen and the USA experienced measles outbreaks again bringing a setback to the global measles elimination goal [20]. At the 63rd World Health Assembly (WHA) in 2010, panel of experts endorsed accelerated measles control targets to be achieved by 2015 [20]. In 2013, a regional goal was established to eliminate measles by the year 2020 by WHO, South-East Asian Region (SEAR) [21]. In 2013, WHO recommended 2 doses of measles vaccine for all children and at least 1 dose prior to international travel for adolescents and adults who were unsure about their immunity status [22]. In 2019, member countries of WHO South-East Asia Region adopted a "Strategic Plan for Measles and Rubella Elimination 2020-2024" to prevent deaths and disabilities caused by these highly infectious childhood killer diseases [23].

\section{Global resurgence of measles}

After decades of progress in measles elimination efforts, the hard fought gains are being threatened by a $31 \%$ increase in the number of measles cases reported globally between 2016 and 2017 [24]. There have been many outbreaks in the United States since the elimination of endemic measles. In 2014, an outbreak of 383 cases was reported in Ohio; between 2014 and 2015, 147cases of measles were reported in California and in 2017, 75 cases were reported in Minnesota. From January 1 to November 7, 2019, 1261 individual cases of measles had been confirmed in 31 states of USA the largest number since 2000 [25]. In Europe, the number of reported cases in 2018 was triple than that in 2017 and 15 times that in 2016 [1]. In addition, it is likely that endemic measles has now been reestablished in several European countries where transmission had previously been interrupted [24]. Current outbreaks include the Democratic Republic of the Congo, Ethiopia, Georgia, Samoa, Ukraine, Kazakhstan, Kyrgyzstan, Madagascar, Myanmar, Philippines, Sudan, Thailand and Ukraine, causing many deaths - mostly among young children. WHO reported 117,075 measles cases and 1205 deaths in Madagascar between October 2018 and April 2019 [1]. An extensive outbreak ravaged in Democratic Republic of Congo in 2019 where close to a quarter of a million people had been infected and nearly 5,000 people died. WHO mentioned the outbreak as the world's largest and fastest-moving epidemic [26].

\section{Measles infection: Bangladesh perspective}

Bangladesh initiated the Expanded Program of Immunization on 7th April, 1979. Single dose measles vaccine for children aged 9 months was introduced in immunization program in 1989 and second dose was administered at 15 months of age since 2012. In 2015, estimated measles routine vaccine national coverage increased up to $92 \%$ for first dose of measles vaccine and $81 \%$ for the second dose. Apart from high routine vaccine coverage nationwide, Bangladesh has implemented the strategy to provide a second opportunity for measles vaccination through supplementary immunization activities. Several other initiatives like strengthening the case-based surveillance 
system, developing and maintaining an accredited measles laboratory network to reach the goal of elimination of measles have been adopted. SIAs were carried out in 2005-2006, 2010, 2014 and 2019. After implementation of nationwide SIAs there was a drastic decline in the occurrence of the disease. In Bangladesh, incidence of measles cases decreased from 40 to 6 per million during 20002016 which constituted to a reduction up to $84 \%$. In 2005-2006, confirmed measles cases dropped to 6 from 14,877 (2005). Unfortunately, the rate of occurrence of the disease was varying in the subsequent years. In 2016, measles cases increased to 972 confirmed cases in Bangladesh [27]. A program assessment was conducted using WHO Programmatic Risk Assessment Tool for measles in 2016 and it was found that 8 districts were at very high risk for measles transmission, 13 districts at high risk, 24 and 19 districts at medium risk and low risk respectively [28].

\section{Challenges in global elimination of measles}

A multitude of factors pose challenges in achieving and maintaining the elimination of measles. It is primarily due to disinclination amongst parents to vaccinate their children, explosive outbreaks of measles in both developed and developing countries and international travelling especially to measles endemic areas [29]. The reason of disinclination amongst the parents to vaccinate their children is based on a conflicting vaccinesafety misinformation which arose by an article published in Lancet demonstrating a link between measles-mumps-rubella (MMR) vaccine and the development of autism in children. Although several studies have now thoroughly debunked that work, it gained attention on some social media networks and continues to be enforced by a small group of anti-vaccine activists [30]. Consequently, there has been a sharp fall in vaccination rates. Another cause behind the growing number of unvaccinated individuals accounts to be the unfamiliarity alongside lack of dread for the outcome of measles infection [31]. In addition, regarding inter-individual transmission dynamics, the fact that one measles virus infected person can be the source to infect 12-18 peoples which makes super spreader part of the picture. Individuals who infect an especially large number of secondary contacts, as compared to most others, are known as super spreaders [32]. The epidemiological concept of the basic reproduction number, $R$ nought $\left(R_{0}\right)$, an indicator of the transmissibility of infectious agents within a population has shed some light to identify people at high risk of contracting an infection and where an outbreak can be effectively intercepted. $R_{0}$ is defined as the average number of secondary cases caused by a primary case in a fully susceptible population. Moreover, an important milestone in regard to $R_{0}$ is that the herd immunity threshold can be determined from it. As $R_{0}$ increases, higher immunization coverage is required to achieve herd immunity. Thus the herd immunity threshold is critical to interrupt transmission in a population and also it can be used as a target for immunization programs to stop the spread of disease [33]. Determinants of $R_{0}$ include the probability of transmission between an infectious individual and a susceptible individual, the type and frequency of contacts between individuals, and the duration of infectivity [34]. Public health researchers frequently use the measles $R_{0}$ range as $12-18$ making it the most contagious of common diseases [35]. Even measles can reappear among vaccinated populations and this finding is pursuant to the observation that in 1989, an explosive school-based outbreak in Finland resulted in 51 cases, several of whom had been previously vaccinated. One child alone infected 22 others. It was noted during this outbreak that when vaccinated siblings shared a bedroom with an infected sibling, seven out of nine became infected as well [36]

Despite measles is a vaccine-preventable disease, high transmissibility, propagated misinformation suggesting that the risk and consequences of measles are inconsequential and the measles vaccine phobia could be the reasons behind widening pockets of unvaccinated children which have created a pathway to the measles outbreaks hitting several countries around the world today.

\section{Conclusion}

In the last couple of years, progress towards measles elimination has stalled and there have been explosive outbreaks around the world. Global 
resurgence of measles virus raises concerns for childhood mortality as well as lifelong disability ranging from brain damage and blindness to hearing loss. People living in urban slums or in remote rural areas, unregistered with health clinics and beyond the reach of health workers are mostly under-immunized and unvaccinated. Identifying and reaching the under-immunized population spans a sequence of essential steps like training health workers, maintaining the cold chain, collecting data and raising awareness of the benefits of vaccination. Precise planning, community-based training and a range of tailored approaches are required to maximize protection against measles infection. Enactment and enforcement of measles elimination initiatives by the governments and partners such as the Measles \& Rubella Initiative, Gavi, the Vaccine Alliance, UNICEF and other organizations are must and thereby the success of vaccination can be back on track worldwide and measles could be the next virus to be wiped out globally.

\section{References}

1. Paules $\mathrm{Cl}$, Marston HD, Fauci AS. Measles in 2019 - going backward. N Engl J. 2019; 380(23): 2185-2187.

2. Plumet S, Duprex WP, Gerlier D. Dynamics of viral RNA synthesis during measles virus infection. J Virol. 2005; 79(11): 6900-6908.

3. Udem SA, Cook KA. Isolation and characterization of measles virus intracellular nucleocapsid RNA. J Virol. 1984; 49(1): 57-65.

4. Griffin DE. Measles virus-induced suppression of immune responses. Immunol Rev. 2010; 236(1): 176-189.

5. Plattet $P$, Alves $L$, Herren $M$, Aguilar $\mathrm{HC}$. Measles virus fusion protein: structure, function and inhibition. Viruses. 2016; 8(4): 112-115.

6. World Health Organization. Nomenclature for describing the genetic characteristics of wildtype measles viruses (update): Part I. Geneva: World Health Organization; 2001.

7. Mosquera MM, de Ory F, Echevarría JE. Measles virus genotyping and circulating genotypes. Open Vaccine J. 2010; 3(1): 76-85.
8. Riddell MA, Rota JS, Rota PA. Review of the temporal and geographical distribution of measles virus genotypes in the prevaccine and postvaccine eras. J Virol. 2005; 2(1): 87-92.

9. Rota PA, Bellini WJ. Update on the global distribution of genotypes of wild type measles viruses. J Infect Dis. 2003; 187(1): 270-276.

10. Patterson CE, Daley JK, Echols LA, Lane TE, Rall GF. Measles virus infection induces chemokine synthesis by neurons. J Immunol. 2003; 171(6): 3102-3109.

11. Buchanan R, Bonthius DJ. Measles virus and associated central nervous system sequelae. Semin Pediatr Neurol. 2012; 19(3): 107-114.

12. Nardone R, Golaszewski S, Trinka E, Tezzon F, Zuccoli G. Acute disseminated encephalomyelitis preceding measles exanthema. J Neurovirol. 2011; 26(12): 1590-1592.

13. Schneider-Schaulies J, Niewiesk S, SchneiderSchaulies S, Meulen VT. Measles virus in the CNS: the role of viral and host factors for the establishment and maintenance of a persistent infection. J Neurovirol. 1999; B(6): 613-622.

14. Perry RT, Halsey NA. The clinical significance of measles: a review. J Infec Dis. 2004; 189 Suppl 1: S4-16

15. Atmar RL, Englund JA, Hammill $H$. Complications of measles during pregnancy. Clin Infect Dis. 1992; 14: 217-226.

16. Manikkavasagan G, Ramsay M. The rationale for the use of measles post-exposure prophylaxis in pregnant women: a review. J Obstet Gynaecol. 2009; 29: 572-575.

17. University of Washington. Measles and the MMR vaccine: recommendations around pregnancy, including the periconception and postpartum periods: Obstetric consensus statement. Washington: University of Washington; 2019.

18. Centers for Disease Control and Prevention. Measles (rubeola): for healthcare professionals. Atlanta: Centers for Disease Control and Prevention; 2018.

19. Odei MP. Measles is in the news yet again. J Family Med Prim Care. 2018; 7(6): 1166-1172. 
20. O'Connor $P$, Jankovic $D$, Muscat $M$, BenMamou M, Reef S, Papania M, et al. Measles and rubella elimination in the WHO Region for Europe: progress and challenges. Clin Microbiol Infect. 2017; 23(8): 504-510.

21. World Health Organization. Global eradication of measles: report by the Secretariat. Geneva: World Health Organization; 2010.

22. World Health Organization. Measles elimination and rubella/congenital rubella syndrome control: Report of a regional consultation, Kathmandu, Nepal, 19-22 February 2013. India: World Health Organization; 2013.

23. World Health Organization. Outbreak news: measles outbreaks in Europe. Geneva: World Health Organization; 2011.

24. World Health Organization. Sustain accelerate innovate: measles elimination and rubella control in the WHO South-East Asia Region. Geneva: World Health Organization; 2018.

25. Dabbagh A, Laws RL, Steulet C, Dumolard L, Mulders $\mathrm{MN}$, Kretsinger $\mathrm{K}$, et al. Progress toward regional measles elimination worldwide, 2000-2017. Morb Mortal Wkly Rep. 2018; 67(47): 1323-1328.

26. Boyd C. MailOnline, 22 November 2019, UK.

27. Khanal $S$, Bohara $R$, Chacko $S$, Sharifuzzaman $M$, Shamsuzzaman $M$, Goodson JL, et al. Progress toward measles elimination Bangladesh, 2000-2016. Morb Mortal Wkly Rep. 2017; 66(28): 753-757.

28. Lam E, Schluter WW, Masresha BG, Teleb N, Brav-Alcántara $P$, Shefer A, et al. Development of a district-level programmatic assessment tool for risk of measles virus transmission. Risk Anal. 2017; 37(6): 1052-1062.

29. Fiebelkorn AP, Redd SB, Gallagher K, Rota PA, Rota J, Bellini W, et al. Measles in the United States during the postelimination era. J Infect Dis. 2010; 202(10): 1520-1528.

30. Taylor B, Miller E, Farrington C, Petropoulos MC, Favot-Mayaud I, Li J, et al. Autism and measles, mumps, and rubella vaccine: no epidemiological evidence for a causal association. Lancet. 1999; 353(9169): 2026 2029.

31. McLaren Jr RA, Stein JL, Minkoff H. Measles: there is no vaccine against vaccine phobia. Am J Perinat. 2019; 15(8): 201-203.

32. Stein RA. Super-spreaders in infectious diseases. Int J Infect Dis. 2011; 15(8): 510-513.

33. Guerra FM, Bolotin S, Lim G, Heffernan J, Deeks $S L$, Li $Y$, et al. The basic reproduction number $\left(R_{0}\right)$ of measles: a systematic review. Lancet Infect Dis. 2017; 17(12): 420-428.

34. Dietz K. The estimation of the basic reproduction number for infectious diseases. Stat Methods Med Res. 1993; 2(1): 23-41.

35. Anderson RM, May RM. Directly transmitted infectious diseases: control by vaccination. Science. 1982; 215(4536): 1053-1060.

36. Paunio $M$, Peltola $H$, Valle $M$, Davidkin I, Virtanen M, Heinonen OP. Explosive schoolbased measles outbreak: intense exposure may have resulted in high risk, even among revaccinees. Am J Epidemiol. 1998; 148(11): 1103-1110. 